\title{
Belt(s) of debris resolved around the Sco-Cen star HIP $67497^{\star, \star \star}$
}

\author{
M. Bonnefoy ${ }^{1}$, J. Milli ${ }^{2}$, F. Ménard ${ }^{1}$, A. Vigan ${ }^{3}$, A.-M. Lagrange ${ }^{1}$, P. Delorme ${ }^{1}$, A. Boccaletti ${ }^{4}$, C. Lazzoni $^{5}$, \\ R. Galicher ${ }^{4}$, S. Desidera ${ }^{5}$, G. Chauvin ${ }^{1}$, J. C. Augereau ${ }^{1}$, D. Mouillet ${ }^{1}$, C. Pinte ${ }^{1}$, G. van der Plas ${ }^{6}$, \\ R. Gratton ${ }^{5}$, H. Beust ${ }^{1}$, and J. L. Beuzit ${ }^{1}$ \\ 1 Univ. Grenoble Alpes, IPAG; CNRS, IPAG, 38000 Grenoble, France \\ e-mail: mickael. bonnefoy@obs.ujf-grenoble.fr \\ 2 European Southern Observatory (ESO), Alonso de Córdova 3107, Vitacura, Casilla 19001, Santiago, Chile \\ 3 Aix-Marseille Université, CNRS, Laboratoire d'Astrophysique de Marseille, UMR 7326, 13388 Marseille, France \\ ${ }^{4}$ LESIA, Observatoire de Paris, CNRS, Université Paris Diderot, Université Pierre et Marie Curie, 5 place Jules Janssen, \\ 92190 Meudon, France \\ 5 INAF-Osservatorio Astronomico di Padova, Vicolo dell'Osservatorio 5, 35122 Padova, Italy \\ ${ }^{6}$ DAS, Universidad de Chile, camino el observatorio 1515 Santiago, Chile
}

Received 13 May 2016 / Accepted 26 September 2016

\begin{abstract}
Aims. In 2015, we initiated a survey of Scorpius-Centaurus A-F stars that are predicted to host warm-inner and cold-outer belts of debris similar to the case of the system HR 8799. The survey aims to resolve the disks and detect planets responsible for the disk morphology. In this paper, we study the F-type star HIP 67497 and present a first-order modelization of the disk in order to derive its main properties.

Methods. We used the near-infrared integral field spectrograph (IFS) and dual-band imager IRDIS of VLT/SPHERE to obtain angulardifferential imaging observations of the circumstellar environnement of HIP 67497. We removed the stellar halo with PCA and TLOCI algorithms. The disk emission was modeled with the GRaTeR code.

Results. We resolve a ring-like structure that extends up to $\sim 450$ mas $(\sim 50 \mathrm{au})$ from the star in the IRDIS and IFS data. It is best reproduced by models of a non-eccentric ring with an inclination of $80 \pm 1^{\circ}$, a position angle of $-93 \pm 1^{\circ}$, and a semi-major axis of $59 \pm 3$ au. We also detect an additional, but fainter, arc-like structure with a larger extension $(0.65 \operatorname{arcsec})$ South of the ring that we model as a second belt of debris at $\sim 130$ au. We detect ten candidate companions at separations $\geq 1$ ". We estimate the mass of putative perturbers responsible for the disk morphology and compare this to our detection limits. Additional data are needed to find those perturbers, and to relate our images to large-scale structures seen with HST/STIS.
\end{abstract}

Key words. techniques: high angular resolution - planetary systems - stars: individual: HIP 67497

\section{Introduction}

The Scorpius-Centaurus OB association (Sco-Cen) is the nearest site of recent massive star formation (de Zeeuw et al. 1999). The proximity $(d=90-200 \mathrm{pc})$ and young age $(\sim 11-17 \mathrm{Myr})$ of Sco-Cen all contribute to making it an excellent niche for direct imaging (DI) searches of planets and disks. The planet imager instruments GPI (Macintosh \& Graham 2008) and SPHERE (Beuzit et al. 2008) have initiated surveys of the circumstellar environment of a few Sco-Cen stars at unprecedented contrasts and angular resolution. They have already resolved several new debris disks around those stars (Currie et al. 2015; Kasper et al. 2015; Kalas et al. 2015; Lagrange et al. 2016; Draper et al. 2016) with wing-tilt and ringed morphologies indicative of the presence of planets.

We initiated a DI survey with SPHERE to image new giant planets and circumstellar disks around a sample of Sco-Cen F5 to A0 stars with infrared excess. The excesses can be modeled by

\footnotetext{
* Based on observations made with ESO Telescopes at the Paranal Observatory under programme ID 097.C-0060(A)

$\star \star$ This work is based on data products produced at the SPHERE Data Center hosted at OSUG/IPAG, Grenoble.
}

2 black-body components, each corresponding to a belt of debris (Chen et al. 2014, hereafter referred to as C14). This architecture is reminiscent of the solar system, and of benchmark systems previously identified by DI such as HR 8799 (Marois et al. 2008, 2010), or HD 95086 (Rameau et al. 2013) which is also part of Sco-Cen.

Over the course of the survey, we resolved a disk around the F0-type star HIP 67497 (HD 120326). This intermediatemass $\operatorname{star}\left(M=1.6 M_{\odot}\right)$ is located at a distance of $107.4_{-8.7}^{+10.4} \mathrm{pc}$ (van Leeuwen 2007) and belongs to the $16 \mathrm{Myr}$ old (Mamajek et al. 2002) Upper Centaurus Lupus sub-group (Mamajek et al. 1999). C14 modeled the infrared excess of the star with two belts of debris: a cold $(127 \pm 5 \mathrm{~K})$ bright $\left(L_{\mathrm{IR}} / L_{*}=\right.$ $\left.1.1 \times 10^{-3}\right)$ belt at 13.9 au and a second colder $(63 \pm 5 \mathrm{~K})$ dimmer $\left(L_{\mathrm{IR}} / L_{*}=1.4 \times 10^{-4}\right)$ belt at $116.5 \mathrm{au}$. The same team produced an alternative model of the excess requiring only one belt located at $8.82 \pm 1$ au (Jang-Condell et al. 2015). Previous observations with adaptive optics systems did not reveal any companion or structure close to HIP 67497 (Janson et al. 2013). Our resolved observations therefore offer the opportunity to better constrain the radial distribution of the dust around this star and to look for the companions responsible for that distribution. 
Table 1. Log of observations, April 6, 2016.

\begin{tabular}{|c|c|c|c|c|c|c|c|c|}
\hline & & IRDIS & IFS & & & & & \\
\hline $\begin{array}{l}\text { UT time } \\
\text { HH:MM }\end{array}$ & Neutral density & $(\mathrm{s}) \quad$ DIT $\times$ NL & $\begin{array}{l}\mathrm{T} \times N_{\mathrm{EXP}} \\
(\mathrm{s})\end{array}$ & $\begin{array}{l}\triangle \mathrm{PA} \\
\left({ }^{\circ}\right)\end{array}$ & $\begin{array}{l}\langle\text { Seeing }\rangle \\
\left({ }^{\prime \prime}\right)\end{array}$ & Airmass & $\begin{array}{l}\tau_{0} \\
(\mathrm{~ms})\end{array}$ & Remarks \\
\hline 04:49 & ND_1.0 & $4 \times 2 \times 1$ & $8 \times 1 \times 1$ & 0.1 & 1.2 & 1.1 & 3.7 & Unsat. PSF \\
\hline $04: 50$ & ND_0.0 & $32 \times 1 \times 1$ & $64 \times 1 \times 1$ & 0.1 & 1.2 & 1.1 & 4.6 & Star center \\
\hline $04: 50$ & ND_0.0 & $32 \times 8 \times 16$ & $64 \times 4 \times 16$ & 37.5 & 1.1 & 1.1 & 3.5 & ADI sequence \\
\hline 06:01 & ND_1.0 & $4 \times 2 \times 1$ & $8 \times 1 \times 1$ & 0.1 & 1.1 & 1.1 & 2.9 & Unsat. PSF \\
\hline $06: 02$ & ND_0.0 & $32 \times 1 \times 1$ & $64 \times 1 \times 1$ & 0.1 & 1.0 & 1.1 & 3.0 & Star center \\
\hline
\end{tabular}

Notes. The seeing is measured at $0.5 \mu \mathrm{m}$. DIT (Detector Integration Time) refers to the individual exposure time per frame. NDIT is the number of individual frames per exposure, $N_{\mathrm{EXP}}$ is the number of exposures, and $\Delta \mathrm{PA}$ to the amplitude of the parallactic rotation.

We present the observations and data in Sect. 2, and the models in Sect. 3. We discuss the morphology of the disk and the existence of putative perturbers (planets) in Sect. 4.

\section{Observations and data reduction}

We observed HIP 67497 on April 6, 2016 with the VLT/SPHERE instrument (Beuzit et al. 2008) operated in IRDIFS mode (Table 1). The mode enables pupil-stabilized observations of the source placed behind an apodized Lyot coronagraph (92.5 mas radius) with the IRDIS (Dohlen et al. 2008) and IFS (Claudi et al. 2008) sub-instruments operated in parallel. IRDIS yielded images of $\sim 11.1 \times 12.4^{\prime \prime}$ centered onto the star in the $\mathrm{H} 2\left(\lambda_{\mathrm{c}}=1.593 \mu \mathrm{m}\right.$, width $\left.=52 \mathrm{~nm}\right)$ and $\mathrm{H} 3\left(\lambda_{\mathrm{c}}=1.667 \mu \mathrm{m}\right.$, width $=54 \mathrm{~nm}$ ) filters (Vigan et al. 2010). The IFS datacubes are made of 39 images covering the $0.96-1.33 \mu \mathrm{m}$ spectral range and a square field-of-view of 1.8 arcsec. For registration purposes (for both sub-instruments before and after the deep exposures) we obtained frames with satellite spots (Star Center) created by a waffle pattern introduced onto the deformable mirror of the instrument. Those frames were used to assess the position of the star behind the coronagraphic mask. We recorded additional non-saturated exposures of the star placed outside of the coronagraphic mask with the neutral density filter ND_1.0 to estimate the flux and position of point sources.

We reduced the IRDIS data with the Data Reduction Handling software (DRH) of SPHERE (Pavlov et al. 2008). The DRH corrected the raw science frames from the bad pixels, subtracted the dark, and recentered the images onto a common origin using the Star Center images. The field rotation of $37.5^{\circ}$ during the sequence of coronagraphic exposures was sufficient to apply the angular differential imaging (ADI) technique and reveal point sources down to $0.1^{\prime \prime}$. We applied a Principal Component Analysis (PCA) algorithm (Soummer et al. 2012) to the H2 and H3 images separately to attenuate the stellar halo. The small number of modes kept in the PCA (10) enabled us to look for extended structures. We used the TLOCI algorithm (Marois et al. 2014) to identify point sources and to confirm the structures.

The IFS data were analysed with a custom pipeline (Vigan et al. 2015, hereafter referred to as V15). This tool subtracted the background, interpolated the bad pixels, removed the instrument crosstalk, and performed a wavelength calibration of the spectra on the 2D raw frames. The DRH was then used to build the cubes from the 2D calibrated frames. We performed a PCA taking advantage of the wavelength coverage and field rotation to look for point sources (V15). We also performed PCA and TLOCI reductions of the IFS data after collapsing each cube in wavelength in order to reveal the (extended, faint) disk structures.

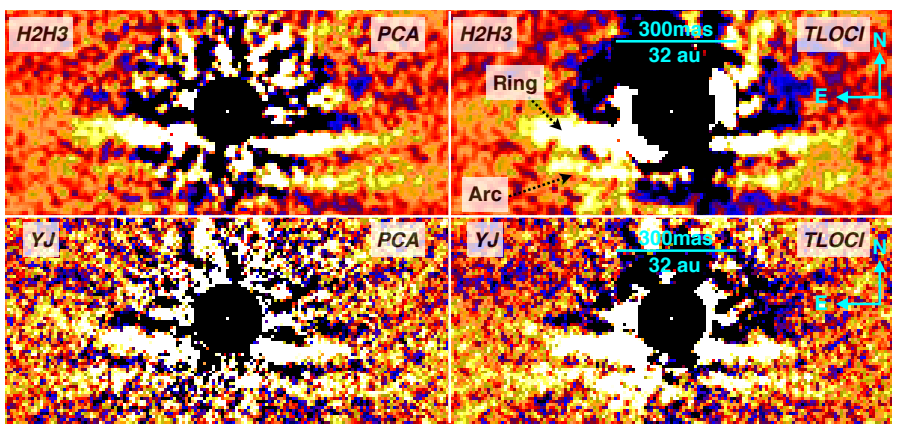

Fig. 1. Images of the disk structure around HIP 67497 obtained with the IFS (YJ), and IRDIS (H2H3), and considering PCA and TLOCI reductions.

We used the True North value of $-1.649 \pm 0.019^{\circ}$, and platescales of $12.257 \pm 0.004$ and $7.46 \pm 0.02 \mathrm{mas} /$ pixel for IRDIS and the IFS, respectively. These values are derived from the observations of the NGC 3603 astrometric field obtained as part of the SPHERE GTO survey on March 30, 2016 (priv. com.).

\section{Disk properties}

We detect an inclined ring at the same position in our collapsed YJ, H2, and H3 images (Fig. 1). The ring structure is seen both in our PCA and TLOCI reductions. It is also retrieved when exploiting both the ADI technique and the wavelength diversity of the IFS and IRDIS (ASDI). We are confident this is a real structure, not related to the stellar halo. The ring extends up to 450 mas, or $50 \mathrm{au}$, from the star in the IRDIS images. It has a smaller apparent extension $(40 \mathrm{au})$ in the YJ images. This is likely due to the lower signal-to-noise ratio $(\mathrm{S} / \mathrm{N})$ of those data. We derived the flux profile (non-corrected from artifacts introduced by the ADI; Milli et al. 2012) along the position angle of the ring from the $\mathrm{H} 2 \mathrm{H} 3$, and YJ PCA images (Fig. 2). To improve the $\mathrm{S} / \mathrm{N}$, we averaged the flux over circular apertures of one FWHM diameter centered on that axis. The error bars for the east and west sides are computed from the dispersion of the flux within each aperture. They are of comparable amplitude. We also report the flux profile of the residual background ("BCK") extracted along the same apertures but tilted at a position angle perpendicular to the disk one (green and pink lines). The profiles indicate that the ring is roughly symmetrical and has a similar flux profile in the YJ and $\mathrm{H} 2 / \mathrm{H} 3$ bands from 15 to $50 \mathrm{au}$. Below $15 \mathrm{au}$ and down to the coronagraph (10 au), the flux profile can be affected by residual speckles. 

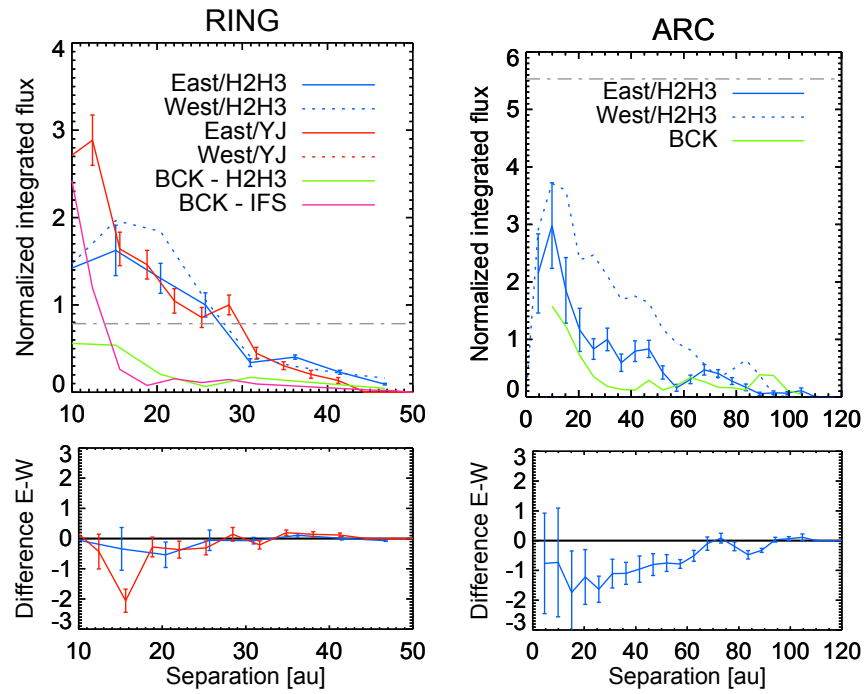

Fig. 2. Radial profile along the disk position angle for the ring $(l e f t)$ and for the arc (right), extracted from the $\mathrm{H} 2 \mathrm{H} 3$, and YJ PCA images and normalized to the median emission of the east side from 15 to $40 \mathrm{au}$. The residuals between the East and West disk profiles are shown at the bottom. The normalization is different for the right and left panels. The dot-dashed line corresponds to the cut of Fig. 1.

An additional arc-like structure ("Arc" in Fig. 1) is detected at 40 mas south of the main ring. It extends visually up to 650 mas on the west side (70 au). This arc structure shows a strong flux asymmetry compared to the ring (Fig. 2). It is also retrieved at the same position into the IFS and IRDIS image for both TLOCI and PCA reductions. As for the ring, we are confident this feature is real and not a residual from the stellar halo. In the following section, we attempt a first characterization of the observed structures to better understand their nature. A more detailed model will be produced in a forthcoming study.

\subsection{Model}

We injected scattered light disk models into the IRDIS data to interpret the observed features. The disk models were generated using the GRaTeR code (Augereau et al. 1999). GRaTeR considers a power-law for the radial distribution of the dust of index $\alpha_{\text {in }}>0$ and $\alpha_{\text {out }}<0$ inward and outward of a reference radius $r_{0}(\theta)$ respectively. The disk model is rotated to the angles of the initial frames, convolved with the measured PSF, and then subtracted from the data. The resulting images are rereduced using the PCA algorithm used for the data reduction to obtain a disc-subtracted image. This step is repeated iteratively by varying the free parameters of the disk model until a reduced $\chi^{2}$ computed in the part of the model-subtracted image in a zone covering the location of the disk on the averaged $\mathrm{H} 2+\mathrm{H} 3$ images, is minimized.

We assumed a gray color for the disk between $\mathrm{H} 2$ and $\mathrm{H} 3$. To limit the parameter space, we adopted a distribution of optically thin dust with constant effective scattering cross-section.

\subsection{Inner ring}

To model the inner ring, we fixed the inner and outer slopes to $\alpha_{\text {in }}=10$ and $\alpha_{\text {out }}=-5$ to mimic sharp edges. We assumed a circular ring, with anisotropic scattering parametrized by a Henyey-Greenstein parameter $g$. Therefore, the model has five free parameters (semi-major axis $r_{0}$, position angle PA,
Table 2. Morphology of the inner belt.

\begin{tabular}{ccccc}
\hline \hline $\mathrm{PA}\left({ }^{\circ}\right)$ & $r_{0}(\mathrm{au})$ & $i_{\text {tilt }}\left(^{\circ}\right)$ & $g$ & $\chi_{\text {region } 1}^{2}$ \\
\hline$-93 \pm 1$ & $58.6 \pm 3$ & $80 \pm 1$ & $0.82 \pm 0.02$ & 2.6 \\
\hline
\end{tabular}

Table 3. Results of the modeling of the belt and arc.

\begin{tabular}{ccccc}
\hline \hline Model & $\alpha_{\text {out }}$ & $r_{0}(\mathrm{au})$ & flux ratio & $\chi_{\text {region 2 }}^{2}$ \\
\hline Two belts & $-8_{-8}^{+4}$ & $130 \pm 8$ & $0.08 \pm 0.03$ & 3.5 \\
Belt+halo & $-1.3_{-0.8}^{+0.6}$ & n.a. & $0.66 \pm 0.40$ & 3.8 \\
\hline
\end{tabular}

inclination $i_{\text {tilt }}, g$, and the flux $)$. The $\chi^{2}\left(\chi_{\text {region } 1}^{2}\right)$ is computed in a region encompassing only the inner belt (see Appendix A). The parameters of the model of best fit are reported in Table 2 and details on the minimization are given in Appendix A. The initial PCA-reduced image, best model and PCA-reduced image after best model subtraction are shown in Fig. 3.

The "arc" structure is still present in the residual image (Fig. 3), thus indicating that it is not a product of self-subtraction effects from the ring. We compare two possible models of that structure in the following section.

\subsection{Model of the arc}

We considered the best model of the inner ring (Sect. 3.2) and modeled the arc with a second, outer belt. We assumed the same inclination, position angle and $g$ parameter as for the inner belt. The outer slope $\alpha_{\text {out }}$, the radius and the flux ratio with respect to the inner belt are left as free parameters. The $\chi^{2}\left(\chi_{\text {region } 2}^{2}\right)$ is now computed on a region encompassing the pixels where the ring and the arc are detected (Appendix A).

As an alternative, we investigated whether a narrow belt and a smooth disk halo could reproduce the ring and the arc. We did so by analogy with HR 8799 where such a halo was proposed (Su et al. 2009). We used for the ring the best fit found in Sect. 3.2 and added a smooth halo modeled by an additional disk of outer slope $\alpha_{\text {out,halo }}<\alpha_{\text {out }}$ and with a flux scaling factor lower than that of the narrow belt. In total, there are three free parameters: $\alpha_{\text {out,halo }}$, the narrow belt flux, and the halo flux.

The best fit parameters are given in Table 3 and the residuals after subtraction of the two models are shown in Fig. 4. The residuals are slightly higher for the case of the ring+halo model. The scenario including two belts of debris is favored by the present data.

\section{Concluding remarks}

The disk around HIP 67497 has an infrared luminosity (IL) in the same range as the disks already resolved around the ScoCen A-F type stars HD 111520, HIP 79977, HD 106906, and HD $115600\left(L_{\mathrm{IR}} / L_{*} \sim 10^{-3}-10^{-4}\right)$. This could explain why we were able to resolve it. For HD 106906, and HD 115600, C14 finds outer belts at shorter separations than observed. In the case of HIP 67497, the second belt corresponds roughly to the location of the coldest belt found by $\mathrm{C} 14$, but the flux ratio between the two belt models found in Sect. 3.3 is of the same order as the ratio of the IL of the belts found by $\mathrm{C} 14$ (7.85).

HST/STIS images of HIP 67497 show extended emission at large scales (Padgett \& Stapelfeldt 2016), which we compare to the SPHERE images in Fig. 5. The inner structures $\left(\leq 3^{\prime \prime}\right)$ 


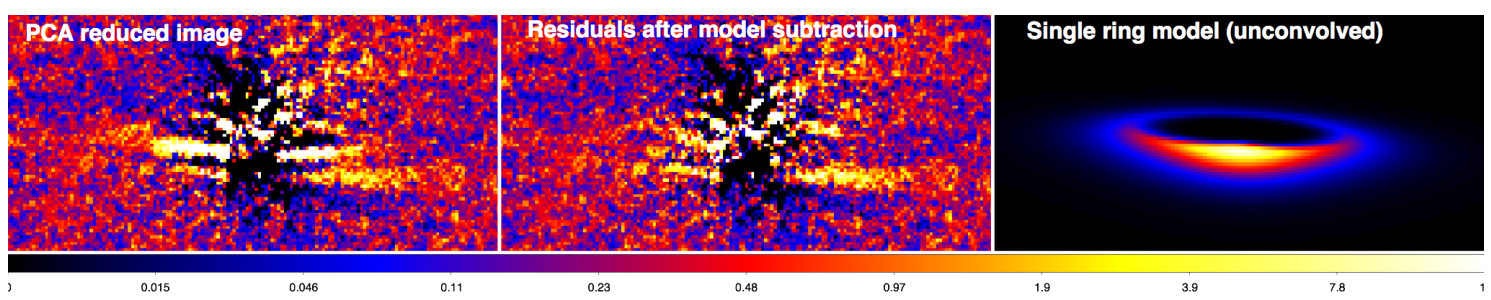

Fig. 3. Results of the forward modeling for the case of a single belt. Left: PCA image of the disk. Middle: Residuals after subtraction of the best single ring model, with the same linear color scale as the left image. Right: best single ring model (unconvolved with the SPHERE PSF) in a log scale to show the fainter backward side of the disk.

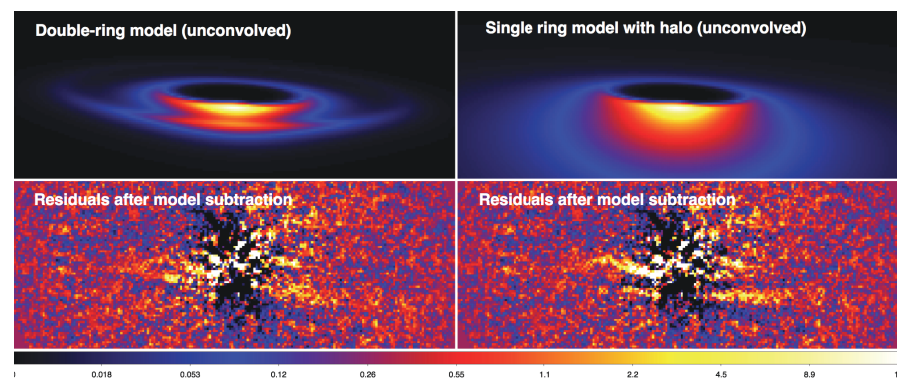

Fig. 4. Results of the forward modeling for the case of two belts (left panels, reduced $\chi^{2}=3.5$ ) and a narrow-ring laid over a smooth halo (right, reduced $\chi^{2}=3.8$ ). The models are shown on top and the postADI residual images at the bottom.

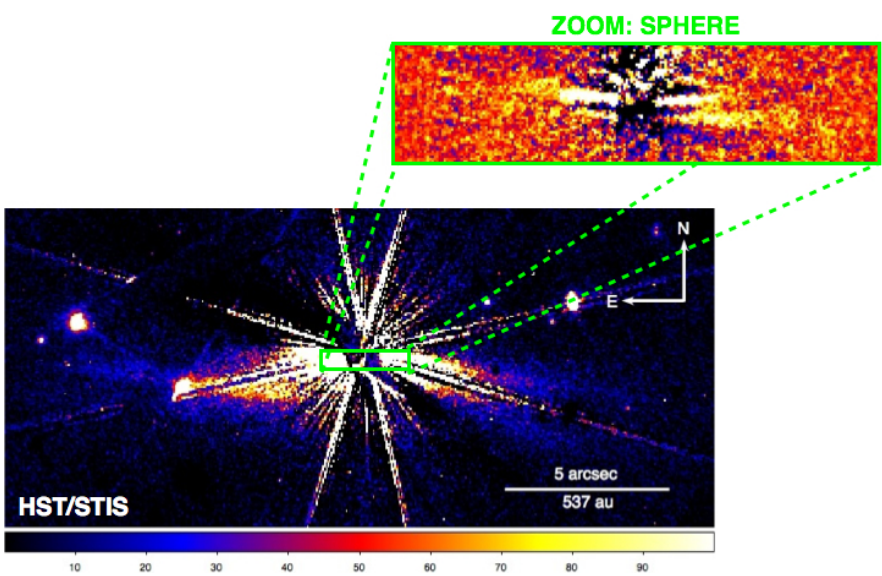

Fig. 5. Sketch showing the HST/STIS and the SPHERE images of the debris disk around HIP 67497.

seen with HST have an orientation compatible with the belt and the arc of the SPHERE images. Another asymmetric feature is seen on the east side up to 8 arcsec, but can not be easily related to the structures seen with SPHERE. Different asymmetries at different scales have already been noticed for HD 106906 (Kalas et al. 2015) and HD 32297 (Esposito et al. 2014; Schneider et al. 2014) for example. One candidate companion (\#1, see Appendix B) could lie within the structures revealed by STIS and the arc seen with SPHERE. The present STIS data are unfortunately affected by blind zones caused by the position of the coronagraphic bars of STIS (only two roll angles).

Several options exist to explain the morphology of the disk of HIP 67497. The observed ring-like structures may be caused by dust-gas interactions (Lyra \& Kuchner 2013). Unfortunately, the gas content of the disk remains unknown. Alternately, planets with different individual eccentricities and semi-major axes (Lee \& Chiang 2016) may also provide an explanation for the double-ring structure. Our observations are sensitive to 1.5 to $15 M_{\text {Jup }}$ in-between the ring and the arc when accounting for the disk inclination (Fig. B.1). We explore in Appendix C the case of one, two, or three perturbers on circular orbit, or one and two planets on eccentric orbits using numerical simulations. The predicted masses can reach $\sim 21 M_{\text {Jup }}$ for the case of a single planet. But the mass estimate of the perturber(s) is sensitive to the eccentricity of the orbit(s). New observations with STIS and SPHERE are required to reveal the full morphology of the disk, improve the detection performances, and to clarify the nature of the CCs.

Acknowledgements. We thank our referee for his/her constructive review of the manuscript. We also thank the ESO staff for gathering those data. We are grateful to the SPHERE consortium for providing instrument platescale and True North. We acknowledge support in France from the ANR (grant ANR-14-CE330018), the PNP, the PNPS, and the Labex OSUG. Gvdp acknowledge support from the Millennium Science Initiative (grant RC130007), and from FONDECYT (grant 3140393)

\section{References}

Augereau, J. C., Lagrange, A. M., Mouillet, D., et al. 1999, A\&A, 348, 557 Baraffe, I., Chabrier, G., Barman, T. S., et al. 2003, A\&A, 402, 701 Beuzit, J.-L., Feldt, M., Dohlen, K., et al. 2008, in SPIE, 7014, 18 Chen, C. H., Mittal, T., Kuchner, M., et al. 2014, ApJS, 211, 25 Claudi, R. U., Turatto, M., Gratton, R. G., et al. 2008, in SPIE, 7014, 3 Currie, T., Lisse, C. M., Kuchner, M., et al. 2015, ApJ, 807, L7 de Zeeuw, P. T., Hoogerwerf, R., de Bruijne, J. H. J., et al. 1999, AJ, 117, 354 Dohlen, K., Langlois, M., Saisse, M., et al. 2008, in SPIE, 7014, 3 Draper, Z. H., Duchêne, G., Millar-Blanchaer, M. A., et al. 2016, ApJ, 826, 147 Esposito, T. M., Fitzgerald, M. P., Graham, J. R., et al. 2014, ApJ, 780, 25 Gladman, B. 1993, Icarus, 106, 247

Jang-Condell, H., Chen, C. H., Mittal, T., et al. 2015, ApJ, 808, 167 Janson, M., Lafrenière, D., Jayawardhana, R., et al. 2013, ApJ, 773, 170 Kalas, P. G., Rajan, A., Wang, J. J., et al. 2015, ApJ, 814, 32 Kasper, M., Apai, D., Wagner, K., \& Robberto, M. 2015, ApJ, 812, L33 Lagrange, A.-M., Langlois, M., Gratton, R., et al. 2016, A\&A, 586, L8 Lee, E. J., \& Chiang, E. 2016, ApJ, 827, 125 Lyra, W., \& Kuchner, M. 2013, Nature, 499, 184 Macintosh, B. A., \& Graham, J. R. 2008, in SPIE, 7015, 701518 Mamajek, E. E., Meyer, M. R., \& Liebert, J. 2002, AJ, 124, 1670 Marois, C., Macintosh, B., Barman, T., et al. 2008, Science, 322, 1348 Marois, C., Zuckerman, B., Konopacky, Q. M., et al. 2010, Nature, 468, 1080 Marois, C., Correia, C., \& Véran, J.-P. 2014, in IAU Proc., 299, 48 Marzari, F. 2014, MNRAS, 442, 1110

Mesa, D., Vigan, A., D’Orazi, V., et al. 2016, A\&A, 593, A119 Milli, J., Mouillet, D., Lagrange, A.-M., et al. 2012, A\&A, 545, A111 Morrison, S., \& Malhotra, R. 2015, ApJ, 799, 41

Mustill, A. J., \& Wyatt, M. C. 2012, MNRAS, 419, 3074 Padgett, D., \& Stapelfeldt, K. 2016, in IAU Proc., 314, 175 Pavlov, A., Feldt, M., \& Henning, T. 2008, in ADASS XVII, 394, 581 Rameau, J., Chauvin, G., Lagrange, A.-M., et al. 2013, ApJ, 779, L26 Schneider, G., Grady, C. A., Hines, D. C., et al. 2014, AJ, 148, 59 Soummer, R., Pueyo, L., \& Larkin, J. 2012, ApJ, 755, L28 Su, K. Y. L., Rieke, G. H., Stapelfeldt, K. R., et al. 2009, ApJ, 705, 314 van Leeuwen, F. 2007, A\&A, 474, 653

Vigan, A., Moutou, C., Langlois, M., et al. 2010, MNRAS, 407, 71 Vigan, A., Gry, C., Salter, G., et al. 2015, MNRAS, 454, 129 Wisdom, J. 1980, AJ, 85, 1122 


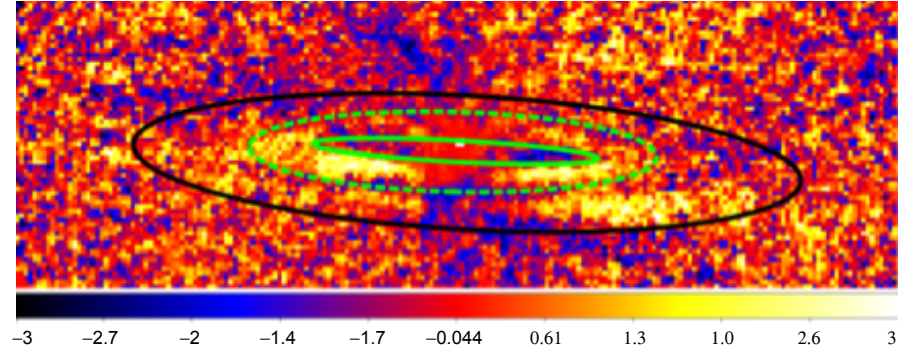

Fig. A.1. Conservative $\mathrm{S} / \mathrm{N}$ map showing the regions used to compute the chi square.
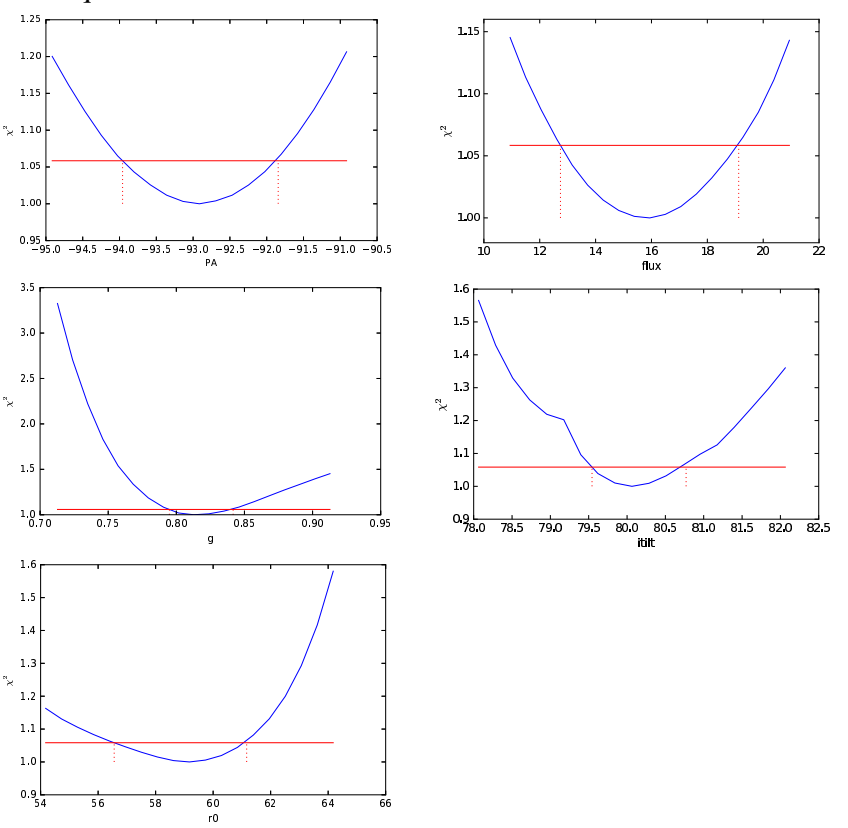

Fig. A.2. Minimization of the free parameters of the single belt model.

\section{Appendix A: Details of the model-fitting}

We report in Fig. A.1 the zones used to compute the $\chi^{2}$ when comparing the disk model to the data. The $\chi^{2}$ for the single belt modeling is computed in the region between the plain green ellipse and the dashed green ellipse. It contains 1041 pixels, or 118 resolution elements. For the case of the model of the outer ring, the $\chi^{2}$ is computed in the region between the plain green ellipse and the black ellipse. It contains 3279 pixels, or 390 resolution elements.

Figure A. 2 shows the $\chi^{2}$ minimization for each of the five free parameters of the single ring model (Sect. 3.2). Figure A.3 shows the $\chi^{2}$ minimization for the free parameters of the models with two belts, or one belt and a halo (Sect. 3.3). The $1 \sigma \chi^{2}$ levels used to estimate the error bars on each parameters are reported in red.

\section{Appendix B: Detected point sources and limits}

We detect ten candidate companions (CC) at large separations in the IRDIS $\mathrm{H} 2$ and $\mathrm{H} 3$ images. Their astrometry and predicted mass, assuming they are bound, are reported in Table B.1. All those point sources lie outside of the field-of-view of the IFS. None of them are aligned with the disk's position angle.

The placement of the candidates in color-magnitude diagrams can help to determine if they have both the luminosity and colors typical of cold substellar companions, or if they are in fact background objects. Such diagrams have been created for the
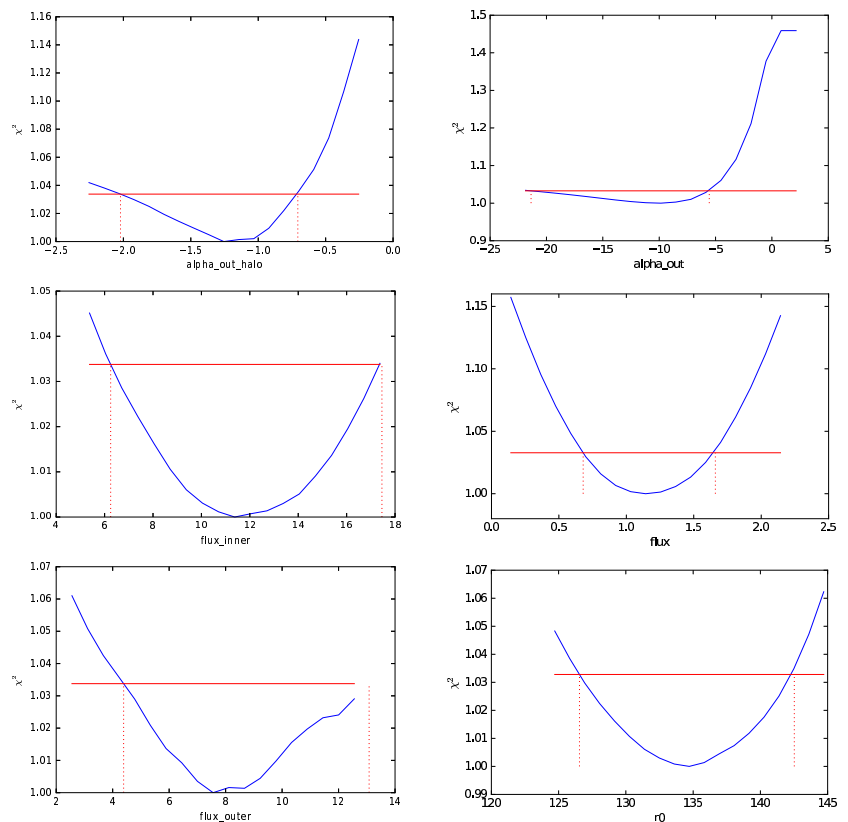

Fig. A.3. Minimization of the free parameters for the model of a single belt and a halo (left column) and of the model with two belts (right column).
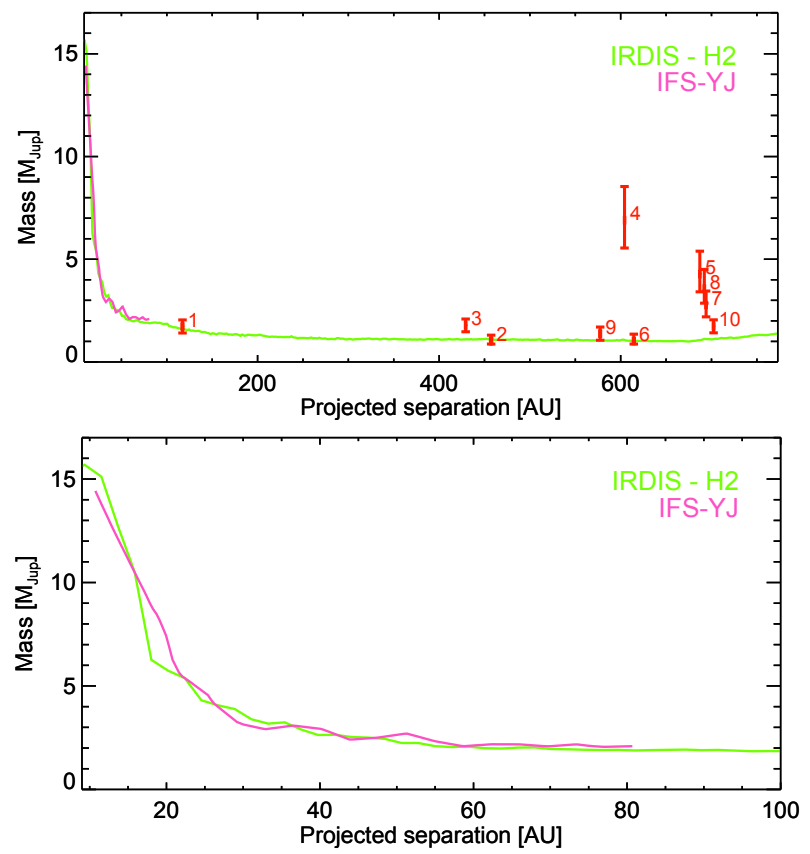

Fig. B.1. Detection limits $(5 \sigma)$ for the IFS (spectral PCA) and IRDIS (TLOCI) converted to mass. The candidate companions are reported in red. Top: full separation range. Bottom: zoom at inner working angles.

IRDIS filters (see a description in Mesa et al. 2016). We show the placement of the CCs of HIP 67497 in Fig. B.2. The CCs \#2, 6 , and 9 have the luminosities typical of T dwarfs but the colors of MK dwarfs, the most common contaminants. This indicates that these CCs are likely to be background objects. All the remaining CCs, except for the CC \#1 and 2, are retrieved in the HST/STIS (optical) images of Padgett \& Stapelfeldt (2016) and are therefore likely background stars.

We estimated the detection limits of the IFS via the injection of fake companions with flat spectra into the datacubes and 


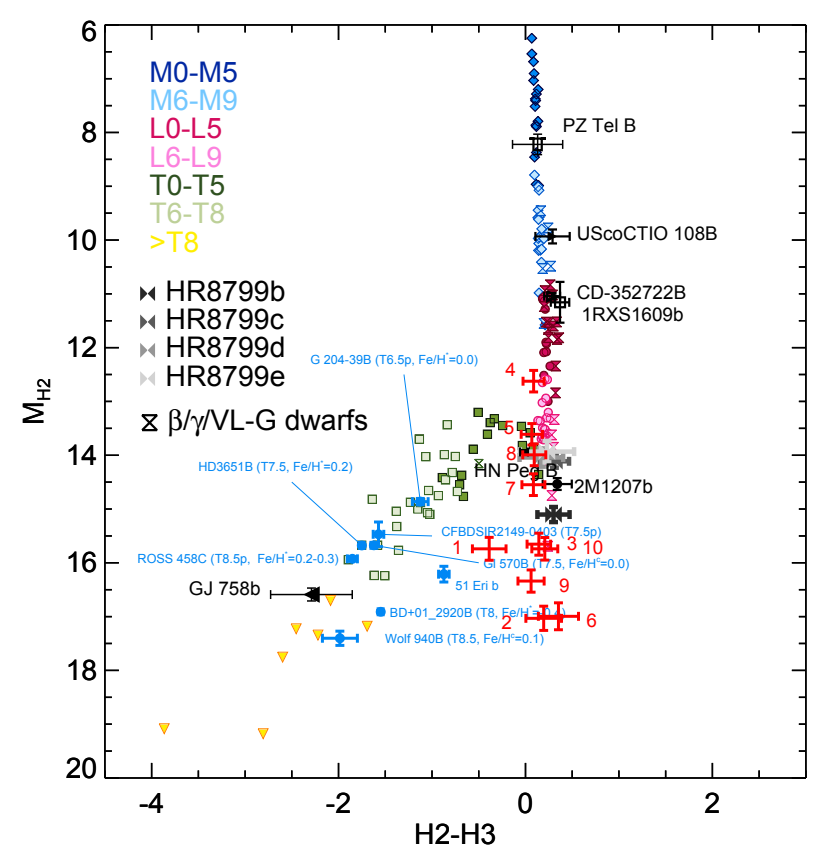

Fig. B.2. Placement of the candidate companions from Table B.1 into color-magnitude diagrams.

Table B.1. Photometry and astrometry of point sources.

\begin{tabular}{llllll}
\hline \hline$\#$ & Band & Contrast & $\begin{array}{l}\text { PA } \\
\left({ }^{\circ}\right)\end{array}$ & $\begin{array}{l}\text { Sep } \\
(\text { mas })\end{array}$ & $\begin{array}{l}M_{\text {Pred }} \\
\left(M_{\text {Jup }}\right)\end{array}$ \\
\hline 1 & H2 & $13.33 \pm 0.09$ & $185.5 \pm 0.3$ & $1092 \pm 6$ & $2 \pm 1$ \\
& H3 & $13.71 \pm 0.13$ & $185.3 \pm 0.4$ & $1088 \pm 8$ & $2 \pm 1$ \\
2 & H2 & $14.62 \pm 0.12$ & $71.9 \pm 0.1$ & $4258 \pm 7$ & $1 \pm 1$ \\
& H3 & $14.42 \pm 0.13$ & $71.9 \pm 0.1$ & $4264 \pm 7$ & $1 \pm 1$ \\
3 & H2 & $13.24 \pm 0.08$ & $293.3 \pm 0.1$ & $3996 \pm 3$ & $2 \pm 1$ \\
& H3 & $13.10 \pm 0.07$ & $293.3 \pm 0.1$ & $3998 \pm 3$ & $2 \pm 1$ \\
4 & H2 & $10.21 \pm 0.07$ & $100.4 \pm 0.1$ & $5626 \pm 2$ & $7 \pm 2$ \\
& H3 & $10.12 \pm 0.06$ & $100.4 \pm 0.1$ & $5628 \pm 2$ & $7 \pm 2$ \\
5 & H2 & $11.20 \pm 0.07$ & $155.8 \pm 0.1$ & $6398 \pm 2$ & $4 \pm 1$ \\
& H3 & $11.13 \pm 0.07$ & $155.8 \pm 0.1$ & $6400 \pm 3$ & $4 \pm 1$ \\
6 & H2 & $14.58 \pm 0.16$ & $279.9 \pm 0.1$ & $5721 \pm 6$ & $1 \pm 1$ \\
& H3 & $14.12 \pm 0.12$ & $280.0 \pm 0.1$ & $5724 \pm 7$ & $1 \pm 1$ \\
7 & H2 & $12.13 \pm 0.07$ & $285.6 \pm 0.1$ & $6462 \pm 3$ & $3 \pm 1$ \\
& H3 & $12.05 \pm 0.08$ & $285.7 \pm 0.1$ & $6458 \pm 3$ & $3 \pm 1$ \\
8 & H2 & $11.58 \pm 0.07$ & $283.7 \pm 0.1$ & $6444 \pm 3$ & $4 \pm 1$ \\
& H3 & $11.48 \pm 0.07$ & $283.7 \pm 0.1$ & $6439 \pm 3$ & $4 \pm 1$ \\
9 & H2 & $13.92 \pm 0.09$ & $351.8 \pm 0.1$ & $5376 \pm 4$ & $2 \pm 1$ \\
& H3 & $13.87 \pm 0.09$ & $351.8 \pm 0.1$ & $5375 \pm 5$ & $2 \pm 1$ \\
10 & H2 & $13.32 \pm 0.09$ & $342.6 \pm 0.1$ & $6539 \pm 5$ & $2 \pm 1$ \\
& H3 & $13.12 \pm 0.08$ & $342.6 \pm 0.1$ & $6541 \pm 4$ & $2 \pm 1$ \\
\hline
\end{tabular}

Notes. The predicted mass if bound to the star is reported in the last column.

used the COND evolutionary tracks to convert the derived contrasts to masses (Baraffe et al. 2003). The IRDIS detection limits were estimated from the TLOCI coefficients and the local noise level. We report those limits in Fig. B.1. The data are sensitive to planetary-mass companions down to $15 \mathrm{au}$.

\section{Appendix C: Putative perturbers}

We investigate the presence of one, two and three giant planets as responsible for the gap between the two possible belts resolved around HIP 67497 using numerical simulations based on published analytical formulae. A detailed description will be made in Lazzoni et al. (in prep.).

If we make the assumption that one planet on a circular orbit only is responsible for the gap, we find that this planet should have a mass of 20.6 $M_{\text {Jup }}$ and semi-major axis $a=90.5$ au following Morrison \& Malhotra (2015). For the case of an eccentric planet, we used the expressions for the half-width of the chaotic zone $\Delta a$ found by Wisdom (1980; for planet eccentricity $\leq 0.3$ ) and Mustill \& Wyatt (2012; for eccentricity $>0.3$ ) as a basis:

$$
\begin{aligned}
& \Delta a=1.3 \times\left(M_{\mathrm{p}} / M_{\text {Star }}\right)^{2 / 7} \times a_{\mathrm{p}}, \\
& \Delta a=1.8 \times\left(M_{\mathrm{p}} / M_{\text {Star }}\right)^{1 / 5} \times e^{1 / 5} \times a_{\mathrm{p}},
\end{aligned}
$$

with $M_{\mathrm{p}}$ the planet mass, $M_{\mathrm{star}}$ the primary star mass, and $e$ the eccentricity of the disk. In our analysis, we consider the planet to arrive at periastron in the nearest point to the inner belt and at apoastron in the nearest point to the outer one. We then substitute in the previous expressions for $\Delta a$, the value of the semi-major axis with the positions of periastron and apoastron in turn. In that case, we predict that planets with low eccentricities $(e=0.13)$ can have masses below our detection threshold.

We also investigated the case of two or three equal-mass planets on circular orbits. We considered planets as near as possible to each other (maximum packing, Gladman 1993) in order to have a stable system but, at the same time, to obtain completely chaotic regions between the planets such that the disk material could not survive in it. For two planets on circular orbits, we followed the results of Marzari (2014) and found that $2.7 M_{\text {Jup }}$ equal-mass perturbers with semi-major axes of 73 and 105 au could clear the gap. For the case of a three planet system with circular orbits, the relations of Marzari (2014) give perturber masses of $0.14 M_{\text {Jup }}$ and semi-major axis of 64.4, 87.5, and $118.7 \mathrm{au}$. We considered a last configuration with two equalmass planets on eccentric orbits, supposing again the maximum packing condition, and following the Eqs. (C.1) and (C.2). We find that low masses $\left(0.1-0.4 M_{\text {Jup }}\right)$ with quite small eccentricities are needed to dig the entire gap.

We recall that multiple assumptions are made here (equal mass pertubers, maximum packing). Therefore, we believe that a more in-depth dynamical analysis of this system is required to understand the morphology of the disk ( $N$-body simulations; beyond the scope of this letter). 